\title{
Transitivity and Chaoticity in 1-D Cellular Automata
}

\author{
Fangyue Chen ${ }^{1}$, Guanrong Chen ${ }^{2}$, Weifeng Jin $^{3}$ \\ ${ }^{1}$ School of Science, Hangzhou Dianzi University, Hangzhou, China \\ ${ }^{2}$ Department of Electronic Engineering, City University of Hong Kong, Hong Kong, China \\ ${ }^{3}$ College of Pharmaceutical Sciences, Zhejiang Chinese Medical University, Hangzhou, China \\ Email: fychen@hdu.edu.cn, eegchen@cityu.edu.hk, jin.weifeng@hotmail.com
}

Received November 22, 2012; revised December 30, 2012; accepted January 14, 2013

\begin{abstract}
Recent progress in symbolic dynamics of cellular automata (CA) shows that many CA exhibit rich and complicated Bernoulli-shift properties, such as positive topological entropy, topological transitivity and even mixing. Noticeably, some CA are only transitive, but not mixing on their subsystems. Yet, for one-dimensional CA, this paper proves that not only the shift transitivity guarantees the CA transitivity but also the CA with transitive non-trivial Bernoulli subshift of finite type have dense periodic points. It is concluded that, for one-dimensional CA, the transitivity implies chaos in the sense of Devaney on the non-trivial Bernoulli subshift of finite types.
\end{abstract}

Keywords: Bernoulli Subshift of Finite Type; Cellular Automata; Devaney Chaos; Symbolic Dynamics; Topological Transitivity

\section{Introduction}

\subsection{Cellular Automata}

Cellular automata (CA), formally introduced by von Neumann in the late 1940s and early 1950s, are a class of spatially and temporally discrete deterministic systems, characterized by local interactions and an inherently parallel form of evolution [1]. In the late 1960s, Conway proposed his now-famous Game of Life, which shows the great potential of CA in simulating complex systems [2]. In the 1980s, Wolfram focused on the analysis of dynamical systems and studied CA in detail [3,4]. In 2002, he introduced the monumental work A New Kind of Science [5]. In fact, mathematical theory of CA was firstly developed by Hedlund about two decades after Neumann's seminal work [6]. Since 2002, Chua et al. provided a rigorous nonlinear dynamical approach to Wolfram's empirical observations [7-10]. All elementary CA (ECA) rules are reorganized into four groups in terms of finite bit stings. There are 40 topologically-distinct period- $k$ rules $(k=1,2,3,6), 30$ topologically-distinct Bernoulli shift rules, 10 complex Bernoulli shift rules, and 8 hyper Bernoulli shift rules. Recently, the dynamical properties of Chua's periodic rules and robust Bernoulli-shift rules with distinct parameters have been investigated from the viewpoint of symbolic dynamics [11-17].

By a theorem of Hedlund [6], a map $f: S^{Z} \rightarrow S^{Z}$ is an one-dimensional cellular automata (1-D CA) if and only if it is continuous and commutes with the shift map $\sigma$. For any 1-D CA, there exists a radius $r>0$ and a local map $\hat{f}: S^{2 r+1} \rightarrow S$ such that

$$
[f(x)]_{i}=\hat{f}\left(x_{[i-r, i+r]}\right),
$$

where notetions will be precisely defined below. In particular, $f$ is an ECA global map when $r=1$ and $S=$ $\{0,1\}$. Each ECA can be expressed by a 3-bit Boolean function and coded by an integer $N$, which is the decimal notation of the output binary sequence of the Boolean function $[5,7,18]$.

\subsection{Definition of Chaos}

Let $X$ be a metric space and $F: X \rightarrow X$ be a continuous map. $F$ is said to be topologically transitive or simply transitive if, for any non-empty open subsets $U$ and $V$ of $X$ there exists a natural number $n$ such that $F^{n}(U) \cap V \neq \varnothing ; F$ is topologically mixing or simply mixing if there exists a natural number $N$ such that $F^{n}(U) \cap V \neq \varnothing$ for all $n \geq N ; F$ is sensitive to initial conditions (or simply sensitive) if there exists a $\delta>0$ such that, for $x \in X$ and for any neighborhood $B(x)$ of $x$, there exists a $y \in B(x)$ and a natural number $n$ such that $d\left(F^{n}(x), F^{n}(y)\right)>\delta$, where $d$ is a distance defined on $X$.

Let $P(F)=\left\{x \in X \mid \exists n>0, F^{n}(x)=x\right\}$ be the set of periodic points of $F, P(F)$ is said to be a dense subset of $X$ if, for any $x \in X$ and any constant $\epsilon>0$, there exists a $y \in P(F)$ such that $d(x, y)<\epsilon$.

Definition 1. $F$ is chaotic on $X$ in the sense of 
Devaney if (1) F is transitive, (2) $P(F)$ is a dense subset of $X$, (3) $F$ is sensitive [19].

It has been proved that additive 1-D CA are chaotic [20]. For general dynamical systems, it has been proved that (1) and (2) together imply (3) [21], and for 1-D CA, (1) implies (3) [22]. In the next section of this paper, it will be proved that, for 1-D CA with Bernoulli subshift of finite type (BSFT), (1) also implies (2).

\subsection{Symbolic Dynamical Systems and SFT}

For a finite alphabet $S=\{0,1, \cdots, k-1\}$, a word over $S$ is a finite sequence $a=\left(a_{0}, \cdots, a_{n-1}\right)$ of elements of $S$. Denote by $S^{n}$ the set of all words of length $n$. If $x$ is a finite or infinite word and $I=[i, j]$ is an interval of integers on which $x$ is defined, then denote

$x_{[i, j]}=\left(x_{i}, \cdots, x_{j}\right)$. Moreover, $a$ is said to be a subword of $x$, denoted as $a \prec x$, if $a=x_{I}$ for some interval $I \subset Z$, where $Z$ is the set of all integers. The set of bi-infinite configurations is denoted by $S^{Z}$, and a distance $d$ on $S^{Z}$ is defined by

$$
d(x, y)=\sum_{i=-\infty}^{+\infty} \frac{\rho\left(x_{i}, y_{i}\right)}{2^{i||}},
$$

where $x=\left(\cdots, x_{-1}, x_{0}, x_{1}, \cdots\right)$,

$y=\left(\cdots, y_{-1}, y_{0}, y_{1}, \cdots\right) \in S^{Z}$, and $\rho\left(x_{i}, y_{i}\right)=0$ if

$x_{i}=y_{i}$, or $=1$ otherwise. It is known that $S^{Z}$ is a compact, perfect and totally disconnected metric space [23].

For a map $f: S^{Z} \rightarrow S^{Z}$, a set $X \subset S^{Z}$ is said to be $f$-invariant if $f(X)=X$. If $X$ is closed and

$f$-invariant, then $(X, f)$ is called a subsystem of the dynamical system $\left(S^{Z}, f\right)$. For example, let $\mathcal{A}$ be a set of some words of length $n$ over $S$, and $\Lambda_{\mathcal{A}}$ be the set of the bi-infinite configurations consisting of all the words in $\mathcal{A}$. Then, $\left(\Lambda_{\mathcal{A}}, \sigma\right)$ is a subsystem of $\left(S^{Z}, \sigma\right)$, where $\sigma$ is the left shift $\left[\sigma_{L}(x)\right]_{i}=x_{i+1}$ (or the right shift $\left.\left[\sigma_{R}(x)\right]_{i}=x_{i-1}\right)$ defined on $S^{Z}$, and $\mathcal{A}$ is called the determinative block system of $\Lambda_{\mathcal{A}}$. The subsystem $\left(\Lambda_{\mathcal{A}}, \sigma\right)$, or simply $\Lambda_{\mathcal{A}}$, is called a subshift of finite type (SFT) with respect to $\mathcal{A}$.

Furthermore, $\Lambda_{\mathcal{A}}$ can be described by a finite directed graph, $G_{\mathcal{A}}=\{\mathcal{A}, E\}$, where each vertex is labeled by a word in $\mathcal{A}$, and $E$ is the set of edges connecting the vertices in $\mathcal{A}$. Two vertices

$a=\left(a_{0}, \cdots, a_{n-1}\right)$ and $b=\left(b_{0}, \cdots, b_{n-1}\right)$ are connected by an edge $\left(a_{0}, \cdots, a_{n-1}\right) \rightarrow\left(b_{0}, \cdots, b_{n-1}\right)$ if and only if $a_{k}=b_{k-1}, k=1,2, \cdots, n-1$. One can think of each element of $\Lambda_{\mathcal{A}}$ as a bi-infinite path on the graph $G_{\mathcal{A}}$. Whereas a directed graph corresponds to a square transition matrix $A=\left(A_{i j}\right)_{m \times m}$ with $A_{i j}=1$ if and only if there is an edge from vertex $b^{(i)}$ to vertex $b^{(j)}$, where $m=|\mathcal{A}|$ is the number of elements in $\mathcal{A}$, and $i$ (or $j$ ) is the code of the corresponding vertex in $\mathcal{A}, i, j=0,1, \cdots, m-1$. Thus, $\Lambda_{\mathcal{A}}$ is precisely defined by the transition matrix $A$.

Remarkably, a $\{0,1\}$ square matrix $A$ is irreducible if, for any $i, j$, there exists an $n$ such that $A_{i j}^{n}>0$; aperiodic if there exists an $n$ such that $A_{i j}^{n}>0$ for all $i, j$, where $A_{i j}^{n}$ is the $(i, j)$ entry of the power matrix $A^{n}$. If $\Lambda_{\mathcal{A}}$ is an SFT of $\left(S^{Z}, \sigma\right)$, then it is transitive if and only if $A$ is irreducible; it is mixing if and only if $A$ is aperiodic. Equivalently, $A$ is irreducible if and only if for every ordered pair of vertices $b^{(i)}$ and $b^{(j)}$ there is a path in $G_{\mathcal{A}}$ starting at $b^{(i)}$ and ending at $b^{(j)}[23,24]$.

\section{Transitivity and Chaoticity}

In this section, it is proved that, for any 1-D CA restricted on its Bernoulli-shift subsystem, the shift transitivity implies the CA transitivity, and transitive nontrivial Bernoulli subshift of finite type (BSFT) has dense periodic points. Consequently, for 1-D CA, transitivity implies chaos in the sense of Devaney on the non-trivial BSFT.

\subsection{Shift Transitivity Implies CA Transitivity}

Definition 2. A closed invariant subset $\Lambda \subset S^{Z}$ of a 1-D CA $f$ is called a Bernoulli-shift subsystem if there exists an integer pair $(q, p)$ with $p r \geq q>0$, such that $f^{p}(x)=\sigma^{q}(x), x \in \Lambda$, where $r$ is the radius of the local map $\hat{f}$ of the CA $f$ and $\sigma$ is the left (or right) shift map.

For simplicity, only consider $\sigma$ as the left shift in the following discussion.

Proposition 1. If $\Lambda$ is a Bernoulli-shift subsystem of a 1-D CA $f$ with $f^{p}(x)=\sigma^{q}(x), x \in \Lambda, p r \geq q>0$, then there exists a $(2 p r+1)$-sequence set $\mathcal{A}$ such that $\Lambda=\Lambda_{\mathcal{A}}=\left\{\left.x \in S^{Z}\right|_{[i-p r, i+p r]} \in \mathcal{A}, \forall i \in Z\right\}$.

Proof: If $\hat{f}: S^{2 r+1} \rightarrow S$ is the local map of $f$, then one can get the $p$ times iteration of $\hat{f}, \hat{f}^{p}: S^{2 p r+1} \rightarrow S$. Thus, $f^{p}(x)=\sigma^{q}(x), x \in \Lambda$, if and only if

$$
\hat{f}^{p}\left(x_{[i-p r, i+p r]}\right)=\left[f^{p}(x)\right]_{i}=\left[\sigma^{q}(x)\right]_{i}=x_{i+q},
$$

for all $i \in Z$. Let

$$
\begin{aligned}
\mathcal{A} & =\left\{\left(a_{0}, \cdots, a_{2 p r}\right) \in S^{2 p r+1} \mid\left(a_{0}, \cdots, a_{2 p r}\right)\right. \\
& \left.=x_{[i-p r, i+p r]}, x \in \Lambda, i \in Z,\right\}
\end{aligned}
$$

and

$$
\Lambda_{\mathcal{A}}=\left\{x \in S^{Z} \mid x_{[i-p r, i+p r]} \in \mathcal{A}, \forall i \in Z\right\},
$$

where $\mathcal{A}$ is a finite set since $|\mathcal{A}| \leq k^{2 p r}$. Then, it follows that $\Lambda=\Lambda_{\mathcal{A}}$. 
Definition 3. The Bernoulli-shift subsystem $\Lambda=\Lambda_{\mathcal{A}}$ in Proposition 1 is called the Bernoulli subshift of finite type (BSFT), and $\mathcal{A}$ is called the determinative block system of $\Lambda$. If BSFT is an infinite set, then it is said to be non-trivial.

Based on Definitions 1, 3 and Proposition 1, an obvious result is the following proposition.

Proposition 2. Consider two BSFTs, $\Lambda_{\mathcal{A}_{1}}$ and $\Lambda_{\mathcal{A}_{2}}$, of a 1-DCA $f$ with

$$
f^{p}(x)=\sigma^{q}(x), x \in \Lambda_{\mathcal{A}_{i}}, i=1,2, p r \geq q>0 .
$$

Then, $\Lambda_{\mathcal{A}_{1}} \subset \Lambda_{\mathcal{A}_{2}}$ if and only if $\mathcal{A}_{1} \subset \mathcal{A}_{2}$.

Theorem 1. Let $\Lambda=\Lambda_{\mathcal{A}}$ be a BSFT of a 1-D CA $f$ with $f^{p}(x)=\sigma^{q}(x), x \in \Lambda$. If the shift $\sigma: \Lambda \rightarrow \Lambda$ is transitive, then $f: \Lambda \rightarrow \Lambda$ is also transitive.

Proof: Since the transitivity of $\sigma$ on $\Lambda$ is equivalent to the existence of a $\tilde{x} \in \Lambda$ such that

$$
\overline{\operatorname{Orb}_{\sigma}(\tilde{x})}=\Lambda \text {, }
$$

where

$$
\operatorname{Orb}_{\sigma}(\tilde{x})=\left\{\tilde{x}, \sigma(\tilde{x}), \sigma^{2}(\tilde{x}), \cdots\right\}
$$

is the orbit of $\sigma$ starting from $\tilde{x}$ and $\overline{\operatorname{Orb}_{\sigma}(\tilde{x})}$ is its closure $[14,15]$. It can be verified that for any $(2 p r+1)$-sequence $\left(a_{0}, a_{1}, \cdots, a_{2 p r}\right) \in \mathcal{A}$, there exists at least an $i \in Z$ such that

$$
\tilde{x}_{[i, i+2 p r]}=\left(a_{0}, a_{1}, \cdots, a_{2 p r}\right) \text {. }
$$

Conversely, for any $i \in Z$, the $(2 p r+1)$-sequence $\tilde{x}_{[i, i+2 p r]} \in \mathcal{A}$.

For the $\tilde{X}$ above, consider the orbit

$$
\operatorname{Orb}_{f}(\tilde{x})=\left\{\tilde{x}, f(\tilde{x}), f^{2}(\tilde{x}), \cdots\right\}
$$

and let $\tilde{\Lambda}=\overline{\operatorname{Orb}_{f}(\tilde{x})}$. It is clear that $\tilde{\Lambda}$ is closed and $f(\tilde{\Lambda})=\tilde{\Lambda}$. Because $\Lambda$ is $f$-invariant and closed, one has $\tilde{\Lambda} \subset \Lambda$ and $f^{p}(x)=\sigma^{q}(x), x \in \tilde{\Lambda}$. Obviously, $f$ is transitive on $\tilde{\Lambda}$.

Let $\tilde{\mathcal{A}}$ denote the determinative block system of $\tilde{\Lambda}$. On one hand, based on Proposition 2 and $\tilde{\Lambda} \subset \Lambda$, one has $\tilde{\mathcal{A}} \subset \mathcal{A}$. On the other hand, since $\tilde{x} \in \tilde{\Lambda}$, it follows that $\tilde{x}_{[i, i+2 p r]} \in \tilde{\mathcal{A}}, \forall i \in Z$, but the $(2 p r+1)$-sequence set consisting of $\tilde{x}_{[i, i+2 p r]}(i \in Z)$ is $\mathcal{A}$. This implies $\mathcal{A} \subset \tilde{\mathcal{A}}$ and $\Lambda \subset \tilde{\Lambda}$. Thus, $\tilde{\Lambda}=\Lambda$, i.e., $f$ is transitive on $\Lambda$. $\square$

\section{Remark 1.}

1) Theorem 1 gives a convenient method to check if a CA $f$ is transitive on a BSFT, since $\sigma$ is transitive on SFT if and only if the transition matrix corresponding to the SFT is irreducible [23,24].

2) Theorem 1 shows that the shift transitivity implies the CA transitivity on the BSFT, but the inverse im- plication may not be correct, with a counter example of ECA $f_{39}$. One has $\left.f_{39}^{2}\right|_{\Lambda}=\left.\sigma\right|_{\Lambda}$, where $\Lambda=\left\{0^{*}, 1^{*}\right\}$ and $0^{*}=(\cdots, 0,0,0, \cdots), 1^{*}=(\cdots, 1,1,1, \cdots)$, so $\Lambda$ contains two points. It is clear that $\left.f_{39}\right|_{\Lambda}$ is transitive but $\left.\sigma\right|_{\Lambda}$ is not. In case BSFT is an infinite set, whether the CA transitivity implies the shift transitivity on the BSFT is still an open problem.

3) When a BSFT $\Lambda$ is a finite set on which $f$ is transitive, then it is a set of $f$-periodic points, $\Lambda=\left\{x, f(x), \cdots, f^{k_{1}}(x)\right\}$ for some $x \in S^{Z}$ and $k_{1}>0$, it is said that $\Lambda$ is trivial.

\section{Remark 2.}

Recall two claims proved in [22]: 1) transitive 1-D CA is always sensitive; 2) a 1-D CA $f$ is transitive but not sensitive on a SFT $\Lambda$ if and only if $\Lambda=\left\{x, \sigma(x), \cdots, \sigma^{k_{2}}(x)\right\}$ for some $x \in S^{Z}$ and $k_{2}>0$. It is easy to be verified that $k_{1}=k_{2}$ and they are common multiples of $p$ and $q$.

\subsection{Transitivity Implies Density of Periodic Points}

Theorem 2 Let $\Lambda=\Lambda_{\mathcal{A}}$ be a BSFT of a 1-D CA $f$ with $f^{p}(x)=\sigma^{q}(x), x \in \Lambda$. If the shift $\sigma: \Lambda \rightarrow \Lambda$ is transitive, then the set of periodic points of $f$, $P(f)=\left\{y \in \Lambda \mid \exists n>0, f^{n}(y)=y\right\}$, is dense in $\Lambda$.

Proof: Let $\Lambda_{\mathcal{A}}$ be the BSFT, and $\mathcal{A}$ be its determinative block system. For any $x \in \Lambda$ and $\epsilon>0$, there exists a positive integer $M(>p r)$ such that

$$
\sum_{i=M+1}^{\infty} \frac{1}{2^{i}}<\epsilon / 2,
$$

and for

$$
\left(a_{0}, \cdots, a_{2 M}\right)=x_{[-M, M]} \prec x \in \Lambda,
$$

it follows that

$$
\left(a_{2 M-2 p r}, \cdots, a_{2 M}\right),\left(a_{0}, \cdots, a_{2 p r}\right) \in \mathcal{A} .
$$

Since $\sigma$ is transitive on $\Lambda$, there exists a path from $\left(a_{2 M-2 p r}, \cdots, a_{2 M}\right)$ to $\left(a_{0}, \cdots, a_{2 p r}\right)$ in the graph $G_{\mathcal{A}}=\{\mathcal{A}, E\}$. Let

$$
\tilde{b}=\left(a_{2 M-2 p r}, \cdots, a_{2 M}, b_{0}, \cdots, b_{k_{0}}, a_{0}, \cdots, a_{2 p r}\right)
$$

be the sequence corresponding to this path. Then, its any $(2 p r+1)$-subsequences belong to $\mathcal{A}$.

Now, construct a cyclic configuration

$$
y=b^{*}=(\cdots, b, b, b, \cdots),
$$

where

$$
b=\left(a_{0}, \cdots, a_{2 M}, b_{0}, \cdots, b_{k_{0}}\right) .
$$

Obviously, $y \in \Lambda$ and $\sigma^{m}(y)=y$, where $m=|b|$ 
is the length of $b$. Thus, $f^{m p}(y)=\sigma^{m q}(y)=y$ and $y_{[-M, M]}=x_{[-M, M]}$, i.e., $y \in P(f)$ and $d(x, y)<\epsilon$. Therefore, the sets of periodic points $P(f)$ is dense in $\Lambda$. $\square$

By Theorem 2 and some results in [21], the following two theorems are obtained.

Theorem 3. If $\sigma$ is transitive on a non-trivial BSFT of a 1-DCA $f$, then $f$ is sensitive on the BSFT.

Theorem 4. A 1-D CA $f$ is chaotic in the sense of Devaney on its transitive non-trivial BSFT.

\subsection{An Example of Transitive ECA Rule}

Rule 26 is a member of Wolfram's class IV and Chua's hyper Bernoulli-shift rules, which defines many subsystems with rich and complex dynamics. This rule's local map is $\hat{f}(0,0,1)=\hat{f}(0,1,1)=\hat{f}(1,0,0)=1$ and $\hat{f}(a, b, c)=0$ for all other triples $(a, b, c) \in\{0,1\}^{3}$, and the corresponding global map is denoted by $f_{26}$.

Proposition 3 There exists a BSFT of $f_{26}$,

$$
\Lambda=\Lambda_{\mathcal{A}}=\left\{\left.x \in S^{Z}\right|_{[i-2, i+2]} \in \mathcal{A}\right\},
$$

such that $f_{26}^{2}(x)=\sigma^{2}(x), x \in \Lambda$, where $S=\{0,1\}$ and

$$
\begin{aligned}
& \mathcal{A}=\{(0,0,0,1,0),(0,0,1,0,1),(0,1,0,0,1), \\
& \\
&(0,1,0,1,1),(0,1,1,0,0),(0,1,1,0,1), \\
&(1,0,0,1,0),(1,0,1,1,0),(1,1,0,0,1),(1,1,0,1,1)\} .
\end{aligned}
$$

Proof: Firstly, $\Lambda$ is a closed set because $S^{Z}-\Lambda$ is an open set. Then, it can be easily verified that $f_{26}(x) \in \Lambda$ for any $x \in \Lambda$, and $\hat{f}_{26}^{2}(a)=a_{i+2}$ for any $a=\left(a_{i-2}, a_{i-1}, a_{i}, a_{i+1}, a_{i+2}\right) \in \mathcal{A}$. This implies that $f_{26}^{2}(x)=\sigma^{2}(x)$ for $x \in \Lambda$.

It is clear that the transition matrix corresponding to $\mathcal{A}$ is

$$
A=\left(\begin{array}{llllllllll}
0 & 0 & 1 & 0 & 0 & 0 & 0 & 0 & 0 & 0 \\
0 & 0 & 0 & 1 & 0 & 0 & 0 & 0 & 0 & 0 \\
0 & 0 & 0 & 0 & 0 & 0 & 1 & 0 & 0 & 0 \\
0 & 0 & 0 & 0 & 0 & 0 & 0 & 1 & 0 & 0 \\
0 & 0 & 0 & 0 & 0 & 0 & 0 & 0 & 1 & 0 \\
0 & 0 & 0 & 0 & 0 & 0 & 0 & 0 & 0 & 1 \\
1 & 1 & 0 & 0 & 0 & 0 & 0 & 0 & 0 & 0 \\
0 & 0 & 0 & 0 & 1 & 1 & 0 & 0 & 0 & 0 \\
0 & 0 & 0 & 0 & 0 & 0 & 1 & 0 & 0 & 0 \\
0 & 0 & 0 & 0 & 0 & 0 & 0 & 1 & 0 & 0
\end{array}\right)
$$

Proposition 4. The transition matrix $A$ is irreducible, so $f_{26}$ is transitive on $\Lambda_{\mathcal{A}}$.

Proof: Let $C=A+I$, where $I$ is the identity matrix, and let $C_{i j}^{n}(1 \leq i, j \leq 10)$ denote the elements of the power matrix $C^{n}$. It can be easily verified that $C_{i j}^{n}>0,1 \leq i, j \leq 10$, for all $n \geq 7$, so $C$ is aperiodic. Recall that a matrix $A$ is irreducible if and only if $A+I$ is aperiodic $[23,24]$. Hence, $A$ is irreducible and so $f_{26}$ is transitive on $\Lambda_{\mathcal{A}}$. $\square$

Theorem $5 \quad f_{26}$ is chaotic in the sense of Devaney on $\Lambda_{\mathcal{A}}$.

\section{Conclusion}

As a particular class of dynamical systems, CA have been widely used for modeling and simulating many physical phenomena. Despite their apparent simplicity, 1-D CA can display rich and complex evolutions, but many properties of their temporal evolutions are undecidable $[25,26]$. Although checking the transitivity based on its definition is very difficult [27], and it alone is not sufficient for chaos to exist in general dynamical systems, this work has rigorously proved that the shift transitivity implies the CA transitivity, and the CA with transitive non-trivial BSFT are chaotic in the sense of Devaney, partly answer the open question whether Devaney chaos in 1-D CA is equivalent to transitivity [28].

\section{Acknowledgments}

This research was jointly supported by the NSFC (Grants No. 11171084 and No. 60872093), the Hong Kong Research Grants Council (Grant No. CityU1117/10) and Foundation of Zhejiang Chinese Medical University (Grant No. 17211076).

\section{REFERENCES}

[1] J. von Neumann, "Theory of Self-Reproducing Automata,” University of Illinois Press, Urbana and London, 1966.

[2] M. Gardner, "The Fantastic Combinations of John Conway's New Solitaire Game Life,” Scientific American, Vol. 223, No. 4, 1970, pp. 120-123. doi:10.1038/scientificamerican1070-120

[3] S. Wolfram, "Computation Theory of Cellular Automata," Communications in Mathematical Physics, Vol. 96, No. 1, 1984, pp. 15-57. doi:10.1007/BF01217347

[4] S. Wolfram, "Theory and Application of Cellular Automata,” Word Scientific, Singapore Cty, 1986.

[5] S. Wolfram, “A New Kind of Science,” Wolfram Media, Inc., Champaign, 2002.

[6] G. A. Hedlund, "Endomorphisms and Automorphism of the Shift Dynamical System,” Mathematical System Theory, Vol. 3, No. 4, 1969, pp. 320-375. doi:10.1007/BF01691062

[7] L. O. Chua, V. I. Sbitnev and S. Yoon, “A Nonlinear Dynamics Perspective of Wolfram's New Kind of Science, Part IV: From Bernoulli-Shift to 1/f Spectrum,” International Journal of Bifurcation and Chaos, Vol. 15, No. 4, 2005, pp. 1045-1183. doi:10.1142/S0218127405012995 
[8] L. O. Chua, V. I. Sbitnev and S. Yoon, “A Nonlinear Dynamics Perspective of Wolfram's New Kind of Scienc, Part VI: From Time-Reversible Attractors to the Arrows of Time,” International Journal of Bifurcation and Chaos, Vol. 16, No. 5, 2006, pp. 1097-1373. doi:10.1142/S0218127406015544

[9] L. O. Chua, J. B. Guan, I. S. Valery and J. Shin, “A Nonlinear Dynamics Perspective of Wolfram's New Kind of Science, Part VII: Isle of Eden,” International Journal of Bifurcation and Chaos, Vol. 17, No. 9, 2007, pp. 28393012. doi:10.1142/S0218127407019068

[10] L. O. Chua, K. Karacs, V. I. Sbitnev, J. B. Guan and J. Shin, "A Nonlinear Dynamics Perspective of Wolfram's New Kind of Science, Part VIII: More Isles of Eden,” International Journal of Bifurcation and Chaos, Vol. 17, No. 11, 2007, pp. 3741-3894. doi:10.1142/S0218127407019901

[11] F. Y. Chen, W. F. Jin, G. R. Chen, F. F. Chen and L. Chen, "Chaos of Elementary Cellular Automata Rule 42 of Wolfram's Class II,” Chaos, Vol. 19, No. 013140, 2009, pp. 1-6.

[12] W. F. Jin, F. Y. Chen, G. R. Chen, L. Chen and F. F. Chen, "Extending the Symbolic Dynamics of Chua's Bernoulli-Shift Rule 56,” Journal of Cellular Automata, Vol. 5, No. 1-2, 2010, pp. 121-138.

[13] W. F. Jin, F. Y. Chen, G. R. Chen, L. Chen and F. F. Chen, "Complex Symbolic Dynamics of Chua's Period-2 Rule 37,” Journal of Cellular Automata, Vol. 5, No. 4-5, 2010, pp. 315-331.

[14] F. F. Chen and F. Y. Chen, "Complex Dynamics of Cellular Automata Rule 119,” Physica A, Vol. 388, No. 6, 2009, pp. 984-990. doi:10.1016/j.physa.2008.12.002

[15] F. F. Chen, F. Y. Chen, G. R. Chen, W. F. Jin and L. Chen, "Symbolics Dynamics of Elementary Cellular Automata Rule 88,” Nonlinear Dynamics, Vol. 58, No. 1-2, 2009, pp. 431-442. doi:10.1007/s11071-009-9490-3

[16] L. Chen, F. Y. Chen, W. F. Jin, F. F. Chen and G. R. Chen, "Some Nonrobust Bernolli-Shift Rules," International Journal of Bifurcation and Chaos, Vol. 19, No. 10, 2009, pp. 3407-3415. doi:10.1142/S0218127409024840

[17] F. Y. Chen, L. Shi, G. R. Chen and W. F. Jin, "Chaos and Gliders in Periodic Cellular Automaton Rule 62,” Journal of Cellular Automata, Vol. 7, No. 4, 2012, pp. 287-302.

[18] J. B. Guan, S. W. Shen, C. B. Tang and F. Y. Chen, "Extending Chua's Global Equivalence Theorem on Wolfram's New Kind of Science," International Journal of Bifurcation and Chaos, Vol. 17, No. 12, 2007, pp. 42454259. doi:10.1142/S0218127407019925

[19] R. L. Devaney, “An Introduction to Chaotic Dynamical Systems,” Addison-Wesley, Hazard, 1989.

[20] P. Favati, G. Lotti and L. Margara, “Additive One-Dimensional Cellular Automata Are Chaotic According to Devaney's Definition of Chaos," Theoretical Computer Science, Vol. 174, No. 1-2, 1997, pp. 157-170. doi:10.1016/S0304-3975(95)00022-4

[21] J. Banks, J. Brooks, G. Cairns, G. Davis and P. Stacey, "On the Devaney's Definition of Chaos," The American Mathematical Monthly, Vol. 99, No. 4, 1992, pp. 332-334. doi:10.2307/2324899

[22] B. Codenotti and L. Margara, "Transitive Cellular Automata Are Sensitive," The American Mathematical Monthly, Vol. 103, No. 1, 1996, pp. 58-62. doi:10.2307/2975215

[23] B. Kitchens, "Symbolic Dynamics: One-Sided, Two-Sided and Countable State Markov Shifts," Springer-Verlag, Berlin, 1998.

[24] Z. L. Zhou, “Symbolic Dynamics,” Shanghai Scientific and Technological Education Publishing House, Shanghai, 1997.

[25] J. Banks, "Regular Periodic Decompositions for Topologically Transitive Maps,” Ergodic Theory and Dynamical Systems, Vol. 17, No. 3, 1997, pp. 505-529. doi:10.1017/S0143385797069885

[26] K. Culik, L. P. Hurd and S. Yu, "Computation Theoretic Aspects of Cellular Automata,” Physica D, Vol. 45, No. 1-3, 1990, pp. 357-378. doi:10.1016/0167-2789(90)90194-T

[27] T. K. S. Moothathu, "Homogeneity of Surjective Cellular Automata," Discrete Continuous Dynamic Systems, Vol. 13, No. 1, 2005, pp. 195-202.

[28] J. Kari, “Theory of Cellular Automata: A Survey,” Theoretical Computer Science, Vol. 334, No. 1, 2005, pp. 3-33. doi:10.1016/j.tcs.2004.11.021 